\title{
The Role of Magnetic Resonance Spectroscopy in Diagnosis of Multiple Sclerosis
}

\author{
Magdy I. Bassiouny, Ahmed A. Torky, Mohamed A. Mansour
}

\begin{abstract}
Department of Radiology, Benha faculty of medicine, Benha University, Egypt.

Correspondence

Mohamed A. Mansour, Department of Radiology, Benha faculty of medicine, Benha University, Egypt.
\end{abstract}

Email:

Mansour10783@gmail.com

Received: 17 May 2020

Accepted: 6 November 2020

\section{Abstract:}

Aim/objective This study aims at emphasizing the role of MRI to: spectroscopy in diagnosis and follow up of post therapeutic response of different stages of the disease of multiple sclerosis. Background Multiple Sclerosis (MS) is a chronic inflammatory, and degenerative autoimmune disorder of the central nervous system (CNS) that is characterized by unpredictable clinical relapses, remissions and by progression of disability over time. There is a need to understand the mechanisms of neurodegeneration in progressive multiple sclerosis. Meanwhile secondary progressive multiple sclerosis develops after an initial relapsing remitting multiple sclerosis course. MRS has the unique ability to characterize the chemical pathology of brain, as well as regions of the brain that are not associated with evident structural abnormalities on conventional MRI. So, it can help to better define the natural history of the disease process and, it can help in monitoring metabolic responses to therapy. Patients and Methods: This study, included 40 patients who presented with clinically diagnosed multiple sclerosis followed by conventional and post contrast MRI and referred for further assessment by MR spectroscopy study. Patients with contraindications to MRI (e.g. cardiac prosthesis, metallic implants and sever claustrophobia) were excluded. Results : The age of included patients ranged from 18 to 45 years with a mean age of $34.67 \pm 8.23$ years. $60 \%$ of the patients were females and $40 \%$ of them were males, the sensitivity, specificity and positive predictive value of H-MRS in the diagnosis of MS were $94.4 \%, 76.2 \%, 71.5 \%$ respectively compared to $87.3 \%, 69.2 \%$, and $65.8 \%$ in case of c-MRI.Conclusion Adding MR spectroscopy to conventional MRI increases 
the specificity and accuracy of MRI study in the diagnosis and follow up post therapeutic response of Multiple sclerosis

Keywords: MRI Spectroscopy, Multiple sclerosis , Relapsing remitting, Primary progressive , Secondary progressive disease

\section{Introduction:}

Multiple Sclerosis (MS) is a chronic inflammatory, demyelinating and degenerative autoimmune disorder of the central nervous system (CNS) characterized by unpredictable clinical relapses and remissions, and by progression of disability over time (1). The incidence of multiple sclerosis has been rising around the world over the last few decades with rising incidence in women, which may support a sex-linked environmental factor. Potential explanations for this include a rise in females' smoking habits, delay age of first pregnancy, declining birth rates, and obesity.

The exact etiology of MS is unknown, but it is widely recognized that it has a complex causation that involves both genetic and environmental factors (2). There is a need to understand the mechanisms of neurodegeneration in progressive multiple sclerosis (3). Secondary progressive multiple sclerosis develops after an initial relapsing remitting multiple sclerosis course (typically 10-15 years), with or without acute exacerbations during the progressive course this results in irreversible and continuous neurological decline (4).

Conventional MRI techniques, such as gadolinium-enhanced T1-weighted (T1W) and T2-weighted (T2W) MRIs, are sensitive indicators of disease activity but do not necessarily provide information about disease pathology or progression.

Correlations between conventional imaging measures and neurological disability measured by the Expanded Disability Status Scale (EDSS) and between imaging and relapse rates have also been modest and variable (i.e., the "clinic-radiological paradox"). This may reflect the poor pathological specificity of conventional MR images, in that the T2W lesion volume "burden of disease" measure does not differentiate between edema, demyelination, axonal loss, and gliosis within apparently normal appearing white 
matter (WM) (5). MRS has its adherents in neurology and the neurosciences and plays a major role in assessment of brain function (6). MRS offers the ability to monitor several pathological processes via their characteristic metabolic markers: N-acetyl aspartate and $\mathrm{N}$-acetylaspartylglutamate (tNAA) for neuroaxonal integrity, creatine and phosphocreatine $(\mathrm{tCr})$ as a putative marker for cell proliferation, choline containing compounds (Cho) involved in membrane turnover, e.g. in the course of inflammation, demyelination and remyelination, myo-Inositol (Ins) for glial (astrocytic) proliferation, glutamate and glutamine (Glx) representing glianeuron metabolism and lactate (Lac) indicating non-oxidative glucose consumption (7).

MRS has the unique ability to characterize the chemical pathology of brain and spinal cord lesions in MS, as well as regions of the brain or spinal cord that are not associated with evident structural abnormalities on conventional MRI. These results in improved diagnosis, it helps to better define the natural history of the disease process and, occasionally, it can help in monitoring metabolic responses to therapy (8). To date, MRS mainly includes single-voxel spectroscopy (SVS) and multi- voxel spectroscopy. SVS has been employed in many previous multiple sclerosis studies. However, abnormalities and impairments are believed to distribute discrepantly in the brain tissue of multiple sclerosis patients. Therefore, SVS is not specific enough to provide a full-scale observation of tissue metabolic changes caused by MS. By contrast, multi-voxel spectroscopy techniques, such as proton 2D magnetic resonance spectroscopic imaging (MRSI), have an advantage in that they provide simultaneous metabolic data across the brain regions, which is critical in the assessment of pathological effects of multiple sclerosis. Therefore, multi-voxel MRSI is increasingly being used in MS studies. In addition, it is clear that the quality of the spectrum, particularly the signal to-noise ratio (SNR) that determines spectral resolution, is largely influenced by magnetic field strength. At high field machines, the improved spectral resolution could increase the sensitivity and accuracy of MRS in evaluating subtle changes in the tissue (9).

\section{Patients and methods:}

This study was a prospective cohort study carried out on clinically diagnosed patients 
with multiple sclerosis at Arab Contractors Medical Center during the period June 2017 - December 2019. The study was approved by the local ethical committee.

PATIENTS: A 40 clinically diagnosed MS patients ranging in age between 18-50 years had been chosen from patients referred from outpatient's neurology clinics. The inclusion criteria include the diagnosis of MS which was determined according to the McDonald criteria and traditional clinical MRI examinations (T2 and FLAIR).

Then patients were classified according to clinical course as having either recurrent relapses with at least one clinical relapse within the last 12 months and noticeable residual symptoms or secondary progressive disease course over a maximum period of two years prior to inclusion in the absence of even discrete relapses after earlier relapsing-remitting disease course. The exclusion criteria was any contra-indications against the MRIscan (e.g. cardiac pacemaker, metal implants, gadolinium allergy, renal impairment and claustrophobia ), A written consent was done for each patient, then underwent full clinical history regarding any neurological attack with revision of the previous radiological examinations ( if available ). MRI scans was performed at Benha university hospital using MRI machine Siemens 1.5 Tesla and Arab contractors medical center .

\section{Conventional MRI protocol:}

MR images comprised axial T1- and T2weighted images as well as fluidattenuated-inversion recovery (FLAIR) images. The sequence parameters $\mathrm{TR} / \mathrm{TE}=7.6 / 3.7 \mathrm{~ms}$ and flip angle $(\mathrm{FA})=8^{\circ}$ for T1-weighted images, 190/80ms for T2weighted images, and TR/TI/TE $=6000 / 2000 / 140 \mathrm{~ms}$ for FLAIR images; field of view $(\mathrm{FOV})=250 \_250 \mathrm{~mm}$; slice thickness $=7.5 \mathrm{~mm}$; and intersection gap $=1 \mathrm{~mm}$.

\section{MR SPECTROSCOPY}

Automatic shimming was employed to compensate for the inhomogeneity of the magnetic field. The line width less than 10 Hz. The signal of water will be suppressed using a chemical shift selective imaging sequence. An outer-volume suppression pulse sequence will be used to avoid signal contamination from outside the volume of interest (VOI). The point resolve spectroscopy sequence (PRESS) technique will be used for VOI selection. The sequence parameters will be TR/TE=2000/53 ms, FOV=150_195mm, slice thickness $=15 \mathrm{~mm}$, in-plane resolution 
$15 \_15 \mathrm{~mm} 2$, and number of averages $=$ 1024.

Automatic shimming was employed to compensate for the inhomogeneity of the magnetic field. The line width will be less than $10 \mathrm{~Hz}$. The signal of water was suppressed using a chemical shift selective imaging sequence (CHESS). An outervolume suppression pulse sequence was used to avoid signal contamination from outside the volume of interest (VOI). The point resolve spectroscopy sequence (PRESS) technique was used for VOI selection. The sequence parameters; TR/ $\mathrm{TE}=2000 / 53 \mathrm{~ms}, \quad \mathrm{FOV}=150195 \mathrm{~mm}$, slice thickness $=15 \mathrm{~mm}$, in-plane resolution 15 $15 \mathrm{~mm} 2$, and number of averages $=1024$. A standard software package was used for post processing the MR spectroscopic data. Metabolite concentrations was measured within individual voxel by calculating the area under the curve.

\section{Imaging analysis}

The review of all conventional and dynamic MRI was performed on the $1.5 \mathrm{~T}$ MR Unit workstation, utilizing the commercially available software (Siemens Medical Systems) by a clinical radiologist with 10 years of experience in crosssectional MRI. Conventional MR images were thoroughly assessed, and lesions were located and characterized according to their number, size, shape, signal criteria and pattern of contrast enhancement. Three user-defined regions of interest (ROIs) were placed on the bases of abnormal FLAIR/T2 signal intensity or enhanced lesions or both. Placement of a control ROI into an apparently normal cerebral white matter was also done.

\section{Results:}

The age of the included patients ranged from 18 to 45 years with a mean age of $34.67 \pm 8.23$ years, $60 \%$ of the patients were females and $40 \%$ of them were males.

The MS clinical subtypes in the studied group as classified by McDonald criteria based on history and clinical bases revealed that $60 \%$ of patients had relapsing remitting subtype (RRMS), $27.5 \%$ of them had primary progressive subtype (PPMS) and $12.5 \%$ of them had secondary progressive subtype (SPMS).

The duration of disease among cases ranged from 2 weeks to 10 years with a mean duration of $4.55 \pm 5.15$ years and that the clinical spectrum included a variety of symptoms in which limb weakness was the most common symptom that occurred in $60 \%$ of patients followed by hemiparesis 

that was found in $42.5 \%$ of patients and then visual troubles in $32.5 \%$ of patients.

The NAA level in chronic lesions was significantly different from that in control matter. However, no significant differences were seen between NAA levels in NAWM and acute lesions relative to normal control white matter. Choline levels were significantly different in NAWM and acute lesions but not in chronic lesions. Creatine levels were found to be somewhat variable across multiple sclerosis areas of interest, with the most difference in acute lesions in relation to control white matter.
Myo-inositol levels were significantly different in NAWM, acute lesions and chronic lesions. Glutamate levels were higher in acute enhancing lesions and NAWM relative to control white matter. No significant differences were noted in glutamate levels in chronic lesions relative to control white matter (fig.1 \& 2).

(table 1).

The sensitivity, specificity and positive predictive value of $\mathrm{H}-\mathrm{MRS}$ in the diagnosis of MS were $94.4 \%, 76.2 \%, 71.5 \%$ respectively compared to $87.3 \%, 69.2 \%$, $65.8 \%$ in case of c-MRI.
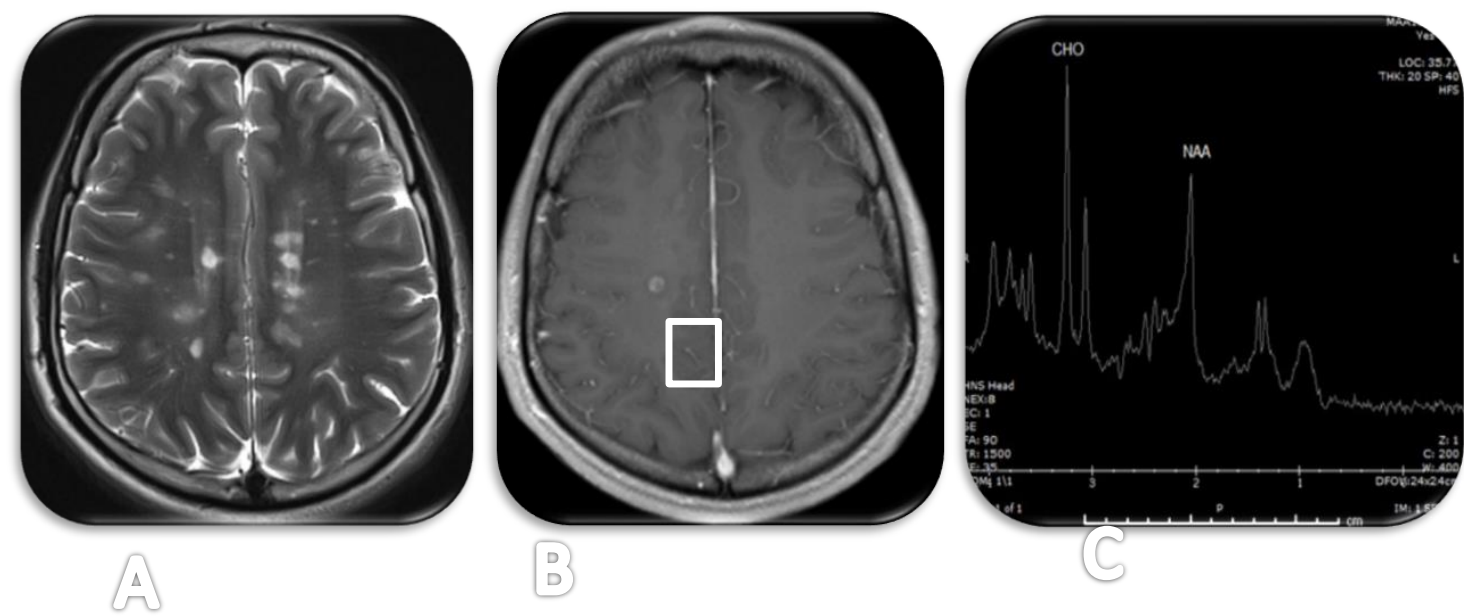

Figure (1): Axial T2 (A) \& post-contrast T1 (B) MRI images, depicting multiple bilateral high fronto-parietal white matter MS plaques, with post- contrast enhancement at right parietal lesion. Figure 1 (C): Magnetic resonance spectroscopy depicting decrease of the NAA peak and increase of the Choline peak together with appearance of lipid peak, impressive of active demyelination. 

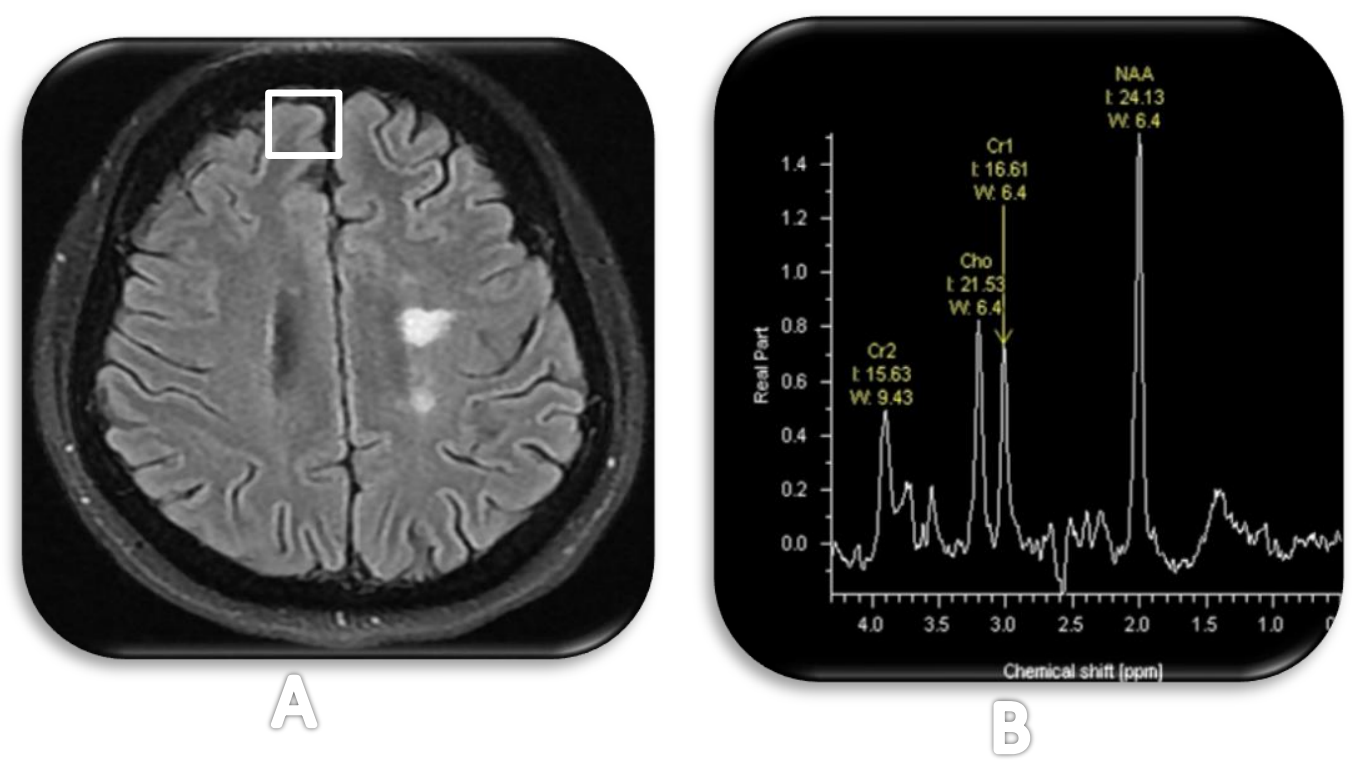

Figure ( 2 ) : A- Axial FLAIR MRI, showing left deep parietal foci of altered MR signal, the largest at the left corona radiata, its axis is perpendicular to the long axis of ventricular system, B- MRS was done, the voxel of concern at left deep parietal region showing mild increase of choline level and subtle decrease of NAA with elevation of creatinine and myo-inositol denoting inactive chronic lesion .

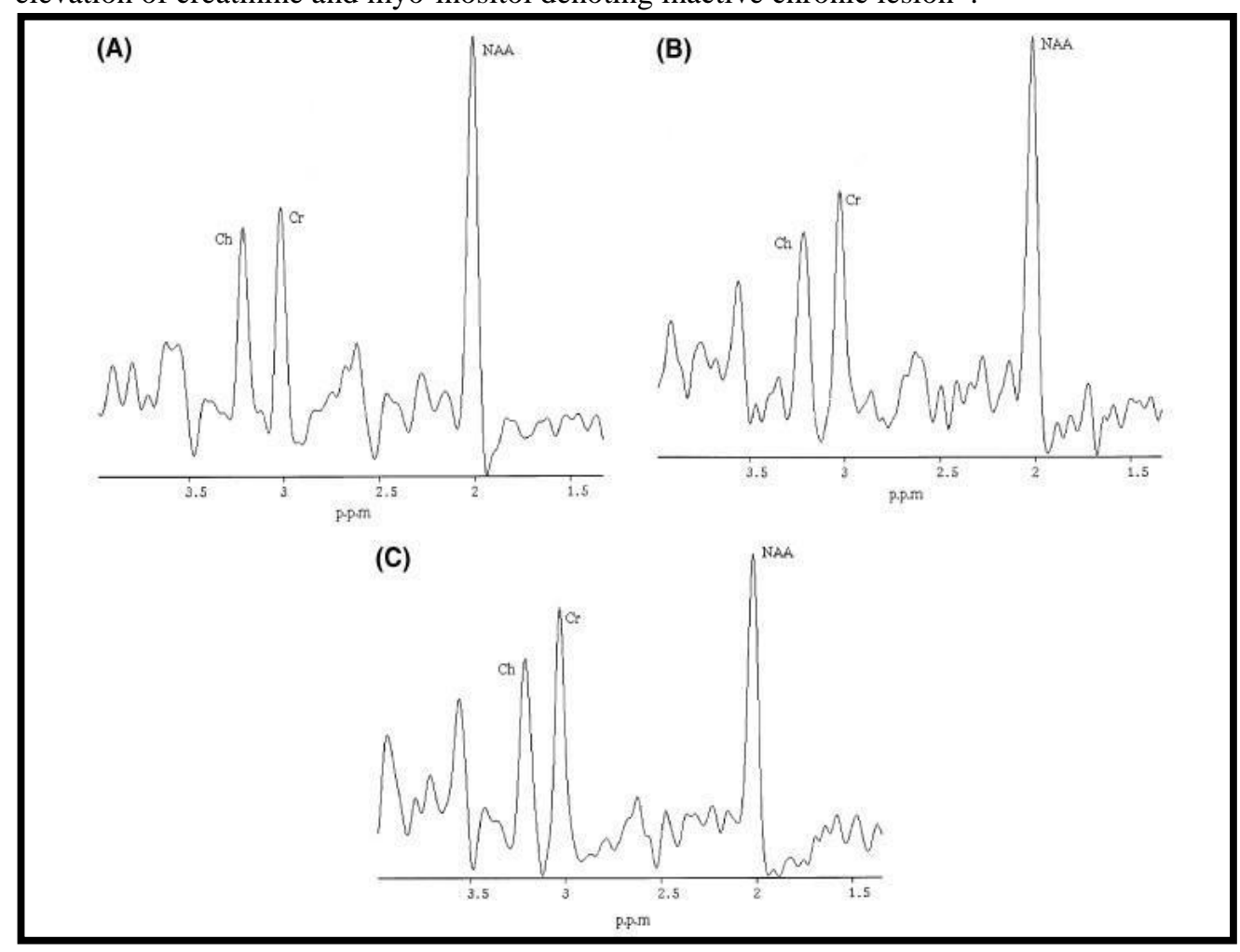

Figure ( 3 ): Comparison between MRS spectra obtained from normal-appearing white matter volumes of interest of a control subject (A), a relapsing-remitting multiple sclerosis patient (B) and a secondary progressive multiple sclerosis patient (C). Ch: Choline; Cr: Creatine; NAA: Nacetyl- aspartate. The NAA peak in both multiple sclerosis patients is reduced than that in the control subject; this decrease is more noted in the progressive form patient. There is no difference observed in the $\mathrm{Ch}$ and $\mathrm{Cr}$ peaks of multiple sclerosis patients and controls. 
Table (1): Comparison of metabolite levels of control white matter, NAWM and regions surrounding acute and chronic lesions

\begin{tabular}{lllllll}
\hline & NAA & Choline & Creatine & Myoinositol & Glutamate & Glutamine \\
\hline $\begin{array}{l}\text { Control white matter } \\
\text { (N= 26) }\end{array}$ & $13.4 \pm 1.7$ & $2.0 \pm 0.4$ & $7.1 \pm 1.2$ & $4.3 \pm 1.2$ & $6.9 \pm 0.8$ & $5.5 \pm 2.0$ \\
NAWN & & & & & $(\mathrm{N}=13)$ & $(\mathrm{N}=7)$ \\
& $12.8 \pm 2.5$ & $2.4 \pm 0.4$ & $8.0 \pm 1.5$ & $5.6 \pm 1.6$ & $8.1 \pm 1.6$ & $6.1 \pm 1.2$ \\
& $\mathrm{P}=1.0$ & $\mathrm{P}=0.03$ & $\mathrm{P}=0.13$ & $\mathrm{P}=0.04$ & $\mathrm{P}=0.03$ & $\mathrm{P}=0.52$ \\
$(\mathbf{N}=\mathbf{2 6})$ & & & & & $(\mathrm{N}=10)$ & $(\mathrm{N}=7)$ \\
Contrast & & & & & & \\
lesions & $12.7 \pm 1.3$ & $2.6 \pm 0.3$ & $8.1 \pm 0.7$ & $6.1 \pm 0.9$ & $8.0 \pm 1.0$ & $6.9 \pm 0.9$ \\
& & & & & & \\
$(\mathbf{N}=\mathbf{1 5})$ & $\mathrm{P}=0.44$ & $\mathrm{P}=0.002$ & $\mathrm{P}=0.02$ & $\mathrm{P}<0.001$ & $\mathrm{P}=0.02$ & $\mathrm{P}=0.12$ \\
Chronic lesions & $10.8 \pm 2.0$ & $2.0 \pm 0.5$ & $7.3 \pm 1.2$ & $5.9 \pm 1.8$ & $6.7 \pm 1.0$ & $5.8 \pm 1.3$ \\
& $\mathrm{P}<0.001$ & $\mathrm{P}=0.94$ & $\mathrm{P}=0.56$ & $\mathrm{P}=0.02$ & $\mathrm{P}=0.77$ & $\mathrm{P}=0.61$ \\
$(\mathbf{N}=\mathbf{2 2})$ & & & & $(\mathrm{N}=11)$ & $(\mathrm{N}=6)$ & $(\mathrm{N}=7)$ \\
\hline
\end{tabular}

\section{Discussion:}

Multiple sclerosis (MS) is a chronic disease which affects the central nervous system, especially the brain, spinal cord and optic nerves, leading to a wide range of symptoms throughout the body. Mild cases may be presented by blurred vision, numbness and tingling in the limbs. And severe cases may experience paralysis, loss of vision and mobility problems, however, this is rare (10). The exact cause of MS is unknown. However, four factors are believed to play a role in the development of the disease. These factors include: the immune system, genetics, environment and infection (11). Interaction of these factors results in damage of the myelin sheath, the protective layer of nerve fibers, resulting in inflammation and scarring thereby disrupting signals between the brain and other parts of the body. The diagnosis of MS requires evidence of demyelination occurring at different times in more than one area of the brain, spinal cord or optic nerves. Diagnostic testing includes: conventional MRI before and after contrast administration to detect active and inactive lesions visual 
evoked potentials test (to analyze electrical activity in the brain), lumbar puncture (to rule out infectious diseases) and blood tests (to exclude other conditions with similar symptoms) (12). Magnetic resonance imaging (MRI) is an important tool in diagnosing MS as well as in monitoring disease activity and progression (13). MRI has revolutionized the investigation, diagnosis and even the treatment of MS and usually, it is the only imaging modality needed for imaging patients with MS and it far surpasses all other tests with respect to its positive predictive value (14). Magnetic resonance spectroscopy (MRS) has the ability to monitor several pathological processes via their characteristic metabolic markers (7). MRS appears capable of depicting changes in white matter that are not detected with routine pulse sequences and its use may prove valuable in monitoring patients after treatment and in formulating their prognosis (15).

The current study revealed that the age of MS patients ranged from 18 to 45 years with a mean age of $34.67 \pm 8.23$ years and that $60 \%$ of the patients were females and $40 \%$ of them were males. This finding comes in line with what was mentioned by Sarbu et al. (16) that the presentation of MS is usually between adolescence and the sixth decade, with a peak at approximately 35 years of age and that the disease shows a strong, well-recognized female predilection with a female: male ratio of about 2:1. Similar higher female to male ratio in MS patients was also reported by Ahlgren et al.(17) who performed their study aiming to determine the population-based nationwide MS prevalence in Sweden, based on observed, registered patients and to investigate the presence of a north-south gradient of MS prevalence. They found that the female to male ratio was $2.35: 1$.

As regards the MS clinical subtypes in MS patients as classified by McDonald criteria based on history and clinical bases, the present study revealed that $60 \%$ of patients had relapsing remitting subtype (RRMS), $27.5 \%$ of them had primary progressive subtype (PPMS) and $12.5 \%$ of them had secondary progressive subtype (SPMS). This finding comes in line with that of the study published by McKay et al. (18) who performed their study aiming to determine risk factors associated with onset of RRMS and PPMS. They found that about $85 \%$ of patients present with relapsing-remitting MS, while $10-15 \%$ present with primary progressive MS. This finding also coincides 
with results of the meta-analysis performed on 21 primary studies in Iran by Kheradmand et al. (19) aiming to estimate the prevalence of different forms and clinical symptoms of MS among Iranian people. They found that relapsing remitting MS was the most common type followed by primary progressive MS and then secondary progressive MS.

The current study revealed that that the duration of disease among cases ranged from 2 weeks to 10 years with a mean duration of $4.55 \pm 5.15$ years and that the clinical spectrum included a variety of symptoms in which limb weakness was the most common symptom that occurred in $60 \%$ of patients followed by hemiparesis that was found in $42.5 \%$ of patients and then visual troubles in $32.5 \%$ of patients.

This finding comes in line with what was reported by Hoang et al.(20) who performed their study aiming to investigate the prevalence of joint contracture (limited passive range of joint motion) and muscle weakness in a population with MS as they found that $56 \%$ of participants had contracture in at least one major joint of upper or lower limb. However, different symptoms were reported by Kheradmand et al.(19) as they mentioned that fatigue was the most common clinical manifestation in MS patients and this difference might be explained by the different ethnicity.

The present study revealed that the $\mathrm{N}$-acetyl aspartic acid (NAA) level was statistically significantly lower $(\mathrm{P}<0.001)$ in chronic lesions than in control matter. However, no significant differences were seen between its levels in normal appearing white matter (NAWM) and acute lesions relative to normal control white matter. The most remarkable MRS findings was reduction in NAA peak in chronic progressive MS and acute MS groups compared to the control group indicating demyelination with neural and axonal damage, while in chronic relapsing MS, NAA showed recovery indicating re-myelination.

Choline levels were significantly higher in NAWM and acute lesions when compared to control matter but not in chronic lesions. This marked increase of choline among acute cases was associated with increase in both lactate and lipid peaks indicating active myelin breakdown, release of cell membrane, inflammatory changes and edema, while among chronic cases, it was increased in progressive form with decreased peak height in relapsing form. Creatine levels were found to be somewhat 
variable across multiple sclerosis areas of interest, with the only statistically significant difference in case of acute enhancing lesions, being higher in relation to control white matter. Relative to control white matter, myo-inositol levels were significantly higher in NAWM, acute lesions and chronic lesions in relation to control white matter and this marked increase in myo-inositol in acute cases indicates glial cell proliferation.

Also, its increase among chronic cases is a sign of gliosis with myelin destruction especially in chronic progressive cases relative to relapsing- remitting cases. Glutamate levels were significantly higher in acute enhancing lesions and NAWM relative to control white matter but no statistically significant differences were noted in glutamate levels in chronic lesions relative to control white matter. Furthermore, no statistically significant differences were found in glutamine levels in NAWN, acute or chronic lesions relative to control white matter. These findings are also nearly similar to those reported by Aboul-Enein et al. (21) who performed their study aiming to define the extent of axonal Inconsistency between our results and results of previous studies might be explained by the degeneration in the NAWM in the remote from focal lesions in patients with RRMS and SPMS. They found that in SPMS, the $\mathrm{NAA} / \mathrm{Cr}$ ratio and absolute concentrations for NAA and Cr were significantly reduced compared to RRMS and to controls and concluded that reduced NAA-levels in the NAWM of patients with MS is a feature of progression. As regards myo-inositol level and similar to our experience, elevated white matter myo-inositol in MS cases compared to controls was reported by Fernando et al.(22) who performed their study aiming to determine NAWM metabolite concentrations in MS patients.

They also reported that creatine level was higher in NAWM compared to controls and that other metabolites did not significantly differ between the MS patients and control subjects. And as for glutamine and glutamate levels, nearly similar results were reported by Tisell et al. (23) who performed their study aiming to find the relationship between disease process in NAWM and the development of white matter lesions. They found that MS patients had higher levels of glutamine and glutamate and reduced levels of NAA compared to healthy controls. 
heterogeneity of MS disease which implies inclusion of patients at different stages of the disease and with different grades of severity.

According to our study, metabolite concentrations were obtained with 3D MRS and the VOI centered mainly on the corpus callosum. In NAWM, the concentration of NAA was observed to be $9 \%$ lower in patients compared to controls. Also, an increase in creatine and choline by $22 \%$ and $32 \%$ respectively was noted. These elevated choline levels differentiated patients from controls with a high sensitivity $94.4 \%$, but less specificity $76.2 \%$, and positive predictive value of $71.5 \%$. Accuracy was improved with the use of proton magnetic resonance spectroscopy in conjunction with conventional MRI, compared to $87.3 \%$ sensitivity, $69.2 \%$ specificity and $65.8 \%$ positive predictive value with using only cMRI. This finding comes in line with what was reported by Shah et al.(24) who performed their study aiming to discuss the clinical characteristics and conventional as well as advanced MRI findings of MS. They mentioned that conventional MRI plays an important role in diagnosing and in monitoring the disease course, however, it lacks sensitivity to gray matter lesions and diffuse damage throughout the white matter and that advanced MRI techniques provide higher specificity and sensitivity to both lesions and normal-appearing gray/white matter contributing in better understanding of MS pathophysiology.

\section{Conclusion}

Magnetic resonance spectroscopy has the ability to monitor several pathological processes in MS and is capable of detecting changes in white matter that are not detected with conventional MRI. That is why its use may prove valuable in monitoring patients after treatment and in formulating their prognosis

\section{References}

1. Dorothea Obert, Gunther Helms, Muriel B. Sättler, Klaus Jung, Benedikt Kretzschmar, Mathias Bähr, et al, : Brain Metabolite changes in Patients with Relapsing-Remitting and Secondary progressive multiple Sclerosis: A Two-Year Follow-Up Study, 2016.

2. Compston A, Coles A: "Multiple sclerosis". Lancet. 2008; 372 (9648): 1502-17.

3. Currie S, Hoggard N, Craven J, Hadjivassiliou $\mathbf{M}$ and Wilkinson $\mathrm{D}$ : Understanding MRI: basic MR physics for physicians Postgraduate Medical Journal, 2013, 89; 209-223; 62(1)17-25.

4. Fox EJ, Rhoades RW: New treatments and treatment goals for patients with relapsing- 
remitting multiple sclerosis. Curr Opin

5. Lublin FD, Reingold SC, Cohen JA, Cutter GR, Sørensen P, Thompson AJ, et al: Defining the clinical course of multiple Sclerosis, The 2013 revisions, Neurology, 2014; 83:278-286.

6. Khan O, Seraji-Bozorgzad N, Bao F, Razmjou S, Caon C, Santiago C, et al : The Relationship Between Brain MR Spectroscopy and Disability in Multiple Sclerosis: 20-Year Data from the U.S. Glatiramer Acetate Extension Study. J Neuroimaging, 2017; 27(1): 97-106.

7. Gülin Öz.PhD, Jeffry R. Alger, MPhil , Peter B.Barker , MPhil, Chris Boesch, MD et al : Clinical Proton MR Spectroscopy in Central Nervous System Disorders , RSNA journal , March 2014; 270(3): 658-679. Published online 2014 Mar. doi: 10.1148/radiol.13130531.

8. Bakshi R, Thompson AJ , Rocca MA , Pelletier D, Dousset V, Frederik Barkhof, et al. MRI in multiple sclerosis: Current status and future prospects . Lancet Neurol. 2008; 7(7):615-25.

9. Gass A , Rocca MA , Agosta F, Ciccarelli O, Chard D, Valsasina P, et al : MRI monitoring of pathological changes in the spinal cord in patients with multiple sclerosis Lancet Neurol, 2015 Apr; 14(4):443-54.

10. 9- Sun J, Song H, Yang Y, Zhang K, Gao X, Li X, et al : Metabolic changes in normal appearing white matter in multiple sclerosis patients using multivoxel magnetic resonance spectroscopy imaging. Medicine (Baltimore) 96(14) (2017) e6534
Neurol, 2012; 25(suppl 1):S11-S19.

11. Brazier Y: Multiple sclerosis : What you need to know . Accessed on 8-2-2020. 2019.

12. Magnifico LA and Ericson C: 4 possible causes of multiple sclerosis (MS). Accessed on 8-2-2020. 2019.

13. Pietrangelo A: Understanding multiple sclerosis (MS). Accessed on 8-2-2020. 2019.

14. Pietrangelo A: MRI images of multiple sclerosis lesions. Accessed on 8-2-2020. 2017.

15. Wijnands JMA, Ekuma O, Kingwell E, Zhu F, Zhao Y, John D Fisk, et al: MRI utilization during the diagnostic and postdiagnostic phases in multiple sclerosis. Mult Scler Relat Disord. 2019; 28:138-44.

16. Henning A , Schar M , Kollias SS , Boesiger $\mathrm{P}$ and Dydak U: Quantitative magnetic resonance spectroscopy in the entire human cervical spinal cord and beyond at 3T. Magn Reson Med. 2008; 59(6):1250-8.

17. Sarbu, N., Shih, R. Y., Jones, R. V , et al (2016). White matter diseases with radiologic-pathologic

correlation. Radiographics, 36(5), 14261447.

18. Ahlgren, C., Odén, A., \& Lycke, J. (2011). High nationwide prevalence of multiple sclerosis in Sweden. Multiple Sclerosis Journal, 17(8), 901-908.

19. McKay, K. A., Tremlett, H., Fisk, J. D., et al , R. A. (2018). Psychiatric comorbidity is associated with disability progression in multiple sclerosis. Neurology, 90(15), e1316-e1323. 
20. Kheradmand, M., Afshari, M., Nasehi, M. M., et al . (2019). Prevalence of subtypes of multiple sclerosis and the most common clinical symptoms in Iranian patients: A metaanalysis. Clinical and Experimental Neuroimmunology, 10(1), 33-40.

21.Hoang, P. D., Cameron, M. H., Gandevia, S. C., et al . (2014). Neuropsychological, balance, and mobility risk factors for falls in people with multiple sclerosis: a prospective cohort study. Archives of physical medicine and rehabilitation, 95(3), 480-486.

22.Aboul-Enein, F., Krššák, M., Höftberger, R., et al. (2010). Reduced NAA-levels in the NAWM of patients with MS is a feature of progression. A study with quantitative magnetic resonance spectroscopy at 3 Tesla. PLoS One, 5(7), e11625.
23.Fernando, K. T. M., McLean, M. A., Chard, D. T., et al . (2004). Elevated white matter myoinositol in clinically isolated syndromes suggestive of multiple sclerosis. Brain, 127(6), 1361-1369.

24. Tisell, A., Leinhard, O. D., Warntjes, J. B. M., et al . (2013). Increased concentrations of glutamate and glutamine in normal-appearing white matter of patients with multiple sclerosis and normal MR imaging brain scans. PLoS One, 8(4), e61817.

25. Shah, M., Xiao, Y., Subbanna, N., Francis, et al . (2011). Evaluating intensity normalization on MRIs of human brain with multiple sclerosis. Medical image analysis, 15(2), 267282

To cite this article: Magdy I. Bassiouny, Ahmed A. Torky, Mohamed A. Mansour. The Role of Magnetic Resonance Spectroscopy in Diagnosis of Multiple Sclerosis, BMFJ 2021; 38 (Radiology):98-111. DOI: 10.21608/bmfj.2021.30484.1267 